\title{
Bucket positioning and its load content during mining in inundated mineral deposit
}

\author{
Nikolay Motyakov, Dmitrii Iakupov, Polina Ivanova and Sergey Ivanov \\ Saint Petersburg Mining University, 2, 21 linija, Vasil'evskij ostrov, Saint Petersburg, 199106, Russia
}

\begin{abstract}
In this paper, we discuss an issue of underwater mining and why modern mining equipment does not fit this purpose. Working in such complicated conditions often has an unsatisfying performance level. We analyzed the processes of mining materials that have not been preliminary dewatered. We also describe variations of positioning system and the importance of their use in underwater mining. A theoretical analysis of excavation process with a perforated bucket has been performed. We present a formula that allows assessing extent of bucket loading by parameters of the working face development. Another formula that allows determining the number of excavation cycles required to complete the bucket loading. A formula for parametric length of working face arc determining by the given stratum depth was introduced. Basing on the acquired formulae, we formulated another one that determines the number of excavation cycles necessary for complete face development for specified cutting parameters. An aggregated graph showing the dependency of the number of excavation cycles on peat shavings width and thickness variables. On the basis of the resulting graph, the best variant for the most complete bucket loading was chosen. In conclusion, we highlighted the peculiarities of underwater mining.
\end{abstract}

\section{Introduction}

After minerals are mined from an inundated working face, they require dehydration which significantly increases costs of production. This is especially relevant for raw peat materials. To comply with requirements listed in the Energy Strategy of Russia for the period until 2035, it is necessary to utilize the best available technologies and the most advanced peat winning systems working in a paradigm of environmentally friendly mining [6]. Currently, existing technologies rarely suit well for underwater mining and, consequently, their performance is at unsatisfying levels when such technologies are used for underwater mining. Most part of peat materials is flushed out from a bucket and the working face itself cannot be visually monitored. Excavated peat mass has a structure of various disruption extents and a water level of $90-92 \%$. In the course of the peat winning process, a significant amount of water is excavated that requires further elimination. The issue of disrupted peat material with a high level of water contents must be resolved step by step [7].

\section{Materials and methods}

The most widespread type of mining machines is an excavator. Its working body is usually constructed of a bucket attached to a boon. It is capable of digging, loading, transferring, unloading and back transferring of mined materials. Screw driven excavators or excavators placed on pontoons can be used for mining in natural peat deposits where no preliminary dewatering was performed [8].

Screw wheelbase construction includes two rotors with flanges that bring a mining machine to motion as the rotors (or Archimedes' screws) rotate. Moving sideward is analogous to a caterpillar-driven machine: a decrease in the torque channeled to one of the rotors allows the mining machine to turn right or left. Screwpropelled machines can traverse trough the toughest offroad environment. Bridges and pontoons allow using mining equipment in inundated deposits. Such platforms can be moved on the water surface of the deposit with a relatively high accuracy. Back shovels have the broadest spectrum of capabilities. High performance of front shovels allows mining materials in a small number of digging cycles. This type of working bodies is suitable for working with materials that are above the excavator standpoint. There are active and passive working bodies. Active bodies have actuating mechanisms in embedded in their construction. These additional mechanisms can be of various designs that depend on the tasks put before the mining machine. A bucket can be attached to arms of various types. The general-purpose (standard) arm is a basic arm that can be adjusted in order to show higher performance in specific working conditions. The telescopic arm extends the reach of the working body. The telescopic construction although decreases the necessity for reallocating the mining machine, it requires better balancing and lubrication of the working body parts. Some special-purpose arms are working bodies themselves, e.g. a ripper arm or a crusher arm $[9,10]$.

\footnotetext{
*Corresponding author: dmitri.yakupov@gmail.com
} 


\section{Results and discussion}

Although modern excavation tools are capable of performing multiple tasks, new requirements set by the developments in the mining industry stress the necessity of designing brand new equipment or new iterations of the currently existing machines and tools suitable, for example, for mining peat materials in inundated natural peat deposits. Bogs and peat deposits not only serve as a source of fossil fuels but also play a key role in economics as natural agricultural systems. Moreover, today, the importance of preserving the natural habitats is also greatly stressed. These factors mean that mining must be not only cost efficient and have high performance but also it must utilize environmentallyfriendly technologies. The most prominent vector of development of the peat mining industry is leveraging the resources of inundated natural peat deposits without their artificial dehydration. Peat excavation can be performed with high efficiency almost in every season. Excavation bears minimal risks of peat fires and eases the process of bog restoration. Moreover, this method excludes discharging of bog waters into the open water intakes that is paralleled with bog dehydration performed prior to working in any peat deposit. A closed cycle of water use allows avoiding excess waste of water. Excavation leaves peat deposits in the state of water reservoirs or bogs can be restored to their initial state. Buckets must be modified specifically for the application in an inundated deposit. Such bucket must be able maintain continuous operation at low or high temperatures and have a long life-cycle. The latter is often achieved by the use of wear-resistant anti-corrosive steels. Well-designed buckets provide maximal performance along with high volume capacity [11, 12].

For example, patent SU 180 8913A1 describes a bucket with a flexible flat plate with several holes. This design has another advantageous feature that is a bucket bottom with ribs that channel the excess water excavated with raw material and thus increase the bucket load capacity. Perforated plate, on the other hand, can lead to losses of the valuable raw material. Patent SU 105 9074A covers the design of a bunker bucket for a trench excavator. The hinged cover plate promotes easier unloading of the excavated raw material and cutting edges of such bucket cut peat shavings of the same size (the best suitable size of shavings must be calculated according to the specific task). This bucket cannot be used for underwater mining, as it is not equipped with additional tools for visual monitoring and removing excess water from the raw material. Patent BY 9977U describes a bucket for drain-laying excavators. This bucket has brackets for chains to attach the bucket to the working body of the excavator. Bucket bottom and side walls are perforated, that promotes excess water drainage. Water flows out without additional mechanical impact, although this design does not allow excess water pressing. Patent BY 4974U describes a peat winning machine mounted on an extended caterpillar platform with a frame and a rotating upper platform. The rotating platform carries the engine, transmission and the cabin. The working body is a multi-bucket frame with a conveyor mounted on the rotating platform. The multibucket frame and the conveyor are positioned perpendicularly with a loading tray in between. This design is highly energy efficient, for layers of milled peat are evenly spread. Nevertheless, this machine cannot be used for underwater mining and has no tools for raw material dehydration. In patent RU 2539508C1, we can find an autonomous machine for underwater mining equipped with a grab bucket with reinforced jaws. It has several obvious advantages: underwater mining applicability, lightweight design, overkeel protection, self-sustainability, applicability regardless of the working depth. Its design includes water drainage channels and a radio beacon that is a part of the positioning system. The high cost of the machine and lack of tools for mechanical dewatering make the use of this excavating tool irrational in most of the tasks. Patent BY $17661 \mathrm{C} 1$ describes a rope scraper bucket for excavation of sapropel. The construction of the bucket includes cover plates actuated by the ropes, leak-proof containers, and skis. The necessity of additional dewatering of the excavated material restricts applicability of this bucket. Nevertheless, this bucket has several advantages: underwater sapropel mining applicability, applicability in conditions of no visual control, high transportability, and easy loading and unloading algorithms. Moreover, controlled cover plates allow accurately selecting the materials to be excavated.

It is necessary to devise a tool with additional active mechanisms that allow pressing the excess water, force unloading, and preventing flushing of extracted peat material. This tool will allow working on a deposit without performing preliminary dewatering. Such a device will reduce the excavation time by 2-3 years and will promote preserving the biological and climate balance of the region. It is highly important to create a tool allowing work in lacking visual control conditions and equipped with a positioning system. Working in an inundated peat deposit goes along with the lack of visual control. That sets stricter requirements for mining tools positioning and may also require workflow automation $[13,14]$.

Taking into account all the information presented in this article, we can conclude that the most rational tool for mining peat materials is a hydraulic excavator with an active back shovel with perforation. The bucket must also have an active mechanism for processing raw peat materials, i.e. performing operations of peat dewatering, pressing, or clearing the bucket from the stuck materials. This excavation tool is best to be placed on a flatbottomed vessel that would serve as a pontoon for mining equipment and other tools necessary for maintaining the mining machinery right on the surface of the peat deposit $[4,15]$.

A bucket movement through the working face is always non-linear and almost always different from a nominal trajectory. The shape and length of this trajectory depend on the strata depth and the volume of a bucket load during the excavation process. Working in an inundated face without visual monitoring of the process increases the chances of inaccurate bucket positioning. Multiple factors, including the proficiency 
of an excavator operator, affect the number of errors made during the excavation process. Hence the effective use of mining equipment requires accurate parameters of a positioning system $[16,17]$.

Accurate positioning can be obtained with various methods and technical means. For example, there is a well-known issue of discrepancy between theoretical and practical values in control units and in units that monitor these theoretical and real values of working body positioning during mineral mining $[18,19]$.

In this case, the use of video technologies can help an excavator operator to monitor the excavation process. Benchmarks can also increase the effectiveness of an excavator. There is also an electro-magnetic positioning that utilizes natural or man-made magnetic fields [20].

Apart from the technologies listed above, we can also enumerate radio, acoustic, probe, satellite, tachymetric, and combined methods for increasing the positioning accuracy. The choice of a method depends on conditions, human-machine factors and economic feasibility [3].

Mathematical models and special-purpose algorithms and their use in the course of the mining process allow matching values of working body speed, its position, and shape and size of the working face $[1,2]$.

We suggest taking a closer look at the process of excavator bucket loading during underwater mining. A special-purposed perforated bucket will serve as a working body. Excavated peat mass will have a disrupted structure. Depending on the extent of disruption, peat materials will have the corresponding degree of loosening $K_{d}(1.25-1.35)$ and the loaded bucket $\mathrm{x}$ can be assessed with a loading coefficient $K_{l}(0.75-$ 0.85 ). The bucket is loaded with peat mass in form of peat shavings that have their size. This parameter affects the structure of the peat mass and determines its ability to release loose water. Depending on this value, we can also determine the length of a bucket movement trajectory for a single excavation cycle [5].

$l$ is shavings length, corresponding to the trajectory length; $b$ is a shavings width that is equal to the width of the bucket cutting edge; $t$ is shavings thickness; $V_{k}$ is a volume of the bucket; and $m$ is a depth of the mined stratum. Shavings length equal to the face length will be:

$$
l=\left(V_{k} K_{l}\right)\left(K_{d} b t\right)^{-1} \text {. }
$$

Arc length $l$ equal to the face length $L_{\mathrm{s}}$ corresponding to the given stratum depth $m$ and known analogue function describing this stratum $f_{s}(m)$ will be:

$$
L_{\mathrm{s}}=\int_{0}^{m} \sqrt{1+\left(f_{\mathrm{s}}^{\prime}(m)\right)^{2}} d x(2) .
$$

Dividing (2) by (1), we get the number of full buckets (excavation cycles) $i$ necessary to complete mining in a face of length $L_{\mathrm{s}}$ with the given shavings width and thickness for the given bucket volume loaded with peat material at its full extent:

$$
i=\frac{b t K_{d} \int_{0}^{m} \sqrt{1+\left(f_{\mathrm{s}}^{\prime}(m)\right)^{2} d x}}{V_{k} K_{l}} .
$$

The arc length of the curve corresponding to the face length can be determined as a function, parameter or polar coordinates. We shall describe two other systems.
The parametric curve of the face for the given stratum depth will be:

$$
L_{\mathrm{s}}=\int_{t_{1}}^{t_{2}} \sqrt{\left(x_{t}^{\prime}(t)\right)^{2}+\left(y_{t}^{\prime}(t)\right)^{2}} d t,
$$

when

$$
\begin{aligned}
& x_{t}=x(t) \\
& y_{t}=y(t) \\
& 0 \leq t \leq m
\end{aligned}
$$

Hence we determine the number of excavation cycles $i$ necessary to complete mining of the whole face length according to the given cutting parameters:

$$
i=\frac{b t K_{d} \int_{0}^{m} \sqrt{\left(x_{t}^{\prime}(m)\right)^{2}+\left(y_{t}^{\prime}(m)\right)^{2}} d t}{V_{k} K_{l}} .
$$

And for the polar coordinate system:

$$
L_{\mathrm{s}}=\int_{\alpha_{1}}^{\beta} \sqrt{(r(\varphi))^{2}+\left(r_{\varphi}^{\prime}(\varphi)\right)^{2}} d \varphi
$$

when

$$
\begin{gathered}
r=r(\varphi) \\
\alpha \leq \varphi \leq \beta .
\end{gathered}
$$

Hence to determine the number of cycles $i$ in the polar coordinates system for the given cutting parameters and the whole length of the face, we use the following formula:

$$
i=\frac{b t K_{d} \int_{\alpha_{1}}^{\beta}\left(\sqrt{(r(\varphi))^{2}+\left(r_{\varphi}^{\prime}(\varphi)\right)^{2}} d \varphi\right.}{V_{k} K_{l}} .
$$

The data for determining the number of cycles for our example are as follows. Stratum depth is $2.5 \mathrm{~m}$; maximum length of working bodies (mining radius) is 10 $\mathrm{m}$; mining depth is $6.6 \mathrm{~m}$; bucket volume is $1 \mathrm{~m}^{3}$. The cutting-edge width is $0.8(0.9 ; 1 ; 1.1 ; 1.2) \mathrm{m}$; loosening coefficient is 1.3 ; loading coefficient is 0.8 ; shavings thickness is $0.1(0.12 ; 0.14 ; 0.16 ; 0.18 ; 0.2) \mathrm{m}$. An arc function for a working face will be parabolic $y=x^{2}$.

Parabolic arc length:

$$
L=\int_{0}^{m} \sqrt{1+\left(y^{\prime}\right)^{2}} d x=\int_{0}^{2.5} \sqrt{1+\left(\left(x^{2}\right)\right)^{2}} d x=\int_{0}^{2.5} \sqrt{1+(2 x)^{2}} d x=\int_{0}^{2.5} \sqrt{1+4 x^{2}} d x
$$

integrated by parts:

$$
L=x \sqrt{1+4 x^{2}}-L+\frac{1}{2} \ln \left|2 x+\sqrt{1+4 x^{2}}\right| .
$$

Thus:

$$
L=\int \sqrt{1+4 x^{2} d x}=\frac{1}{2} x \sqrt{1+4 x^{2}}+\frac{1}{4} \ln \left|2 x+\sqrt{1+4 x^{2}}\right|
$$

by using the Newton-Leibniz formula, we can calculate the arc length value:

$$
L=\left[\frac{1}{2} x \sqrt{1+4 x^{2}}+\frac{1}{4} \ln \left|2 x+\sqrt{1+4 x^{2}}\right|\right]_{0}^{2.5} \approx 6,95 \mathrm{~m} .
$$

This value corresponds to the integral value of the expression (2) given the desired conditions. Complementing this expression with the values of shavings thickness, cutting-edge width, bucket volume, 
coefficient of loosening and coefficient of loading, we can calculate the required number of digging cycles given the values of shavings thickness and cutting edge width. Calculated values are presented in Table 1.

Table 1. The number of digging cycles with full loading of a $1 \mathrm{~m}^{3}$ bucket required for completing the development of a working face with a length $L_{s}$.

\begin{tabular}{|c|c|c|c|c|c|c|c|}
\hline & \multicolumn{6}{|c|}{ Shavings Thickness $t, \mathrm{~m}$} \\
\hline & & 0.1 & 0.12 & 0.14 & 0.16 & 0.18 & 0.2 \\
\hline \multirow{5}{*}{ 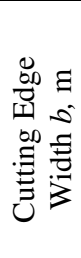 } & 0.8 & 0.903 & 1.08 & 1.26 & 1.45 & 1.63 & 1.81 \\
\hline & 0.9 & 1.02 & 1.22 & 1.42 & 1.62 & 1.83 & 2.03 \\
\hline & 1.0 & 1.13 & 1.35 & 1.58 & 1.81 & 2.03 & 2.26 \\
\hline & 1.1 & 1.24 & 1.49 & 1.73 & 1.98 & 2.24 & 2.48 \\
\hline & 1.2 & 1.35 & 1.61 & 1.91 & 2.17 & 2.44 & 2.71 \\
\hline
\end{tabular}

The table demonstrates that only six values are appropriate for use in the determined conditions. These values are given in bold and are underlined. These values correspond to the buckets with the cutting edge width of $0.8 \mathrm{~m}$ and $0.9 \mathrm{~m}$ that cut the shavings of $0.1 \mathrm{~m}$ and 0.12 $\mathrm{m}$ thickness. In cases of shavings thickness values equal to $0.16 \mathrm{~m}, 0.18 \mathrm{~m}$, or $0.2 \mathrm{~m}$, cut with the cutting edges that are $1.1 \mathrm{~m}, 1.0 \mathrm{~m}$, and $0.9 \mathrm{~m}$ in width respectively, it requires two runs of the working face development. Moreover, the second run will require more precise bucket positioning. The estimated performance of the excavator is almost the same in all six cases.

To determine the cutting parameters of an excavator bucket and to test the theoretical calculations in practice for their further introduction to the real working body prototypes, it is required to perform experiments with the use of models of the mining equipment. The authors are now devising the model of the working equipment that will allow cutting peat shavings using both front and back shovels, whereas the operating load will be created with the help of additional load samples. Another point that bears significant practical importance is the loading capabilities of a bucket working in an inundated deposit. Such experiments are also being devised by the authors. The results of the experiments are to be published in future.

\section{Conclusion}

As of today, no modern technologies allow excavate peat materials without preliminary dewatering and that is why it is necessary to perform a very scrupulous analysis to find the most suitable mining system. To comply with the requirements for excavated peat material quality, it is necessary to use a positioning system that can eliminate so-called 'blind spots' and increase the performance and efficiency of the mining process. Formulae (1) and (2) allow determining the bucket parameters and the parameters of the cut shavings that, being applied, lead to the optimal bucket filling and to the development of the working face in the minimal number of excavation cycles. This leads to an increase in the effectiveness of the mining equipment use.

In this work, we determined the rational geometric parameters of shavings that can help to increase the effectiveness of underwater mining.

Calculations presented in Table 1 demonstrate that the rational use of mining equipment, especially in conditions of lack of visual monitoring, requires accurate parameters of peat shavings cutting acquired with the help of an accurate positioning system.

\section{References}

1. M. M. Hasan, Analytical Framework for Field Positioning and Work Planning for a Backhoe Excavator (2018)

2. K. Matsumura, M. Ito, C. Raima, S. Saiki, Y. Yamazaki, Y. Kurita, ISARC. Proc. of the Int. Symposium on Automation and Robotics in Construction 37, 547-551 (2020)

3. X. Zhang, S. Qiao, L. Quan, L. Ge, IEEE Access, 7, (2019)

4. D.V. Robertson, D.D. Jaeger, R. Eidenberger, System for Excavator Dipper Control (2019)

5. X. Yu, X. Pang, Z. Zou, G. Zhang, Y. Hu, J. Dong, H. Song, Mathematical Problems in Engineering (2019)

6. V.B. Gorokhova, Teoria i Praktika Conf. 2, 405411 (2020)

7. D.R. Iakupov, S.L. Ivanov, P.V. Ivanova, E.K. Permiakova, IOP Conference Series: Materials Science and Engineering 966 (2020)

8. A.A. Naumenko, Transactions of the Krylov State Research Centre S. J. 2, 239-247 (2019)

9. A.I. Markovnina, Innovative development of Lifting and Transport equipment Conf. P., 37$46(2020)$

10. V.S. Velikanov, J. of Mining Institute, 241, 29-36 (2020)

11. V.V Savinkin, V.N. Kusnetsova, Education. Transport. Innovations. Construction. Conf. P. , 61-66 (2020)

12. A.A. Korneev, V.P. Pavlov, Technological Equipment for the Mining And Oil And Gas Industry Conf. P., 58-61 (2017)

13. G.G. Buryy, V.S. Scherbakov, V.S. Skobelev, V.F. Kovalevsky, Vestnik SibADI 16(3(67)), 202213 (2019)

14. A.Yu. Cheban, Earth and Subsurface Use Science 44(1), 56-62 (2021)

15. A.P. Komissarov, I.V. Teliman, Technological equipment for the Mining and Oil and Gas industry Conf. P., 121-125 (2019)

16. P.A. Osipov, A.L. Karyakin, Ural Mining School - Regionam Int. Conf. P., 315 (2018)

17. E.A. Kremcheev, J. of Mining Institute 231, 225234, (2018)

18. S.N. Zharikov, J. of Mining Institute 229, 56-61 (2018)

19. I.A. Korolyov, S.A. Lavrenko, Int. J. of Applied Engineering Research 9 (12), 2031-2037 (2017) 
20. K.R. Argimbaev, D.N. Ligotskiy, M.O. Bovduy, K.V. Mironova, Mining informational and analytical bulletin 5, 35-42 (2018) 